\title{
Heavy-Flavor contribution to Bhabha scattering
}

Bonciani, R ; Ferroglia, A ; Penin, A A

\begin{abstract}
We evaluate the last missing piece of the two-loop QED corrections to the high-energy electronpositron scattering cross section originating from the vacuum polarization by heavy fermions. The calculation is performed within a new approach applicable to a wide class of perturbative problems with mass hierarchy. The result is crucial for the high-precision physics program at existing and future e(+) e(-) colliders.
\end{abstract}

DOI: https://doi.org/10.1103/PhysRevLett.100.131601

Other titles: Two-Loop Heavy-Flavor Contribution to Bhabha Scattering

Posted at the Zurich Open Repository and Archive, University of Zurich

ZORA URL: https://doi.org/10.5167/uzh-13581

Journal Article

Accepted Version

Originally published at:

Bonciani, R; Ferroglia, A; Penin, A A (2008). Heavy-Flavor contribution to Bhabha scattering. Physical Review Letters, 100(13):131601.

DOI: https://doi.org/10.1103/PhysRevLett.100.131601 
ALBERTA-THY-18-07

IFIC/07-66

ZU-TH 26/07

hep-ph/yymmnnn

\title{
Two-Loop Heavy-Flavor Contribution to Bhabha Scattering
}

\author{
R. Bonciani ${ }^{a, *}$, A. Ferroglia ${ }^{b, \dagger}$, and A.A. Penin ${ }^{c, d, \ddagger}$ \\ a Departamento de Física Teòrica, IFIC, CSIC - Universidad de Valencia, \\ E-46071 Valencia, Spain \\ b Institut für Theoretische Physik, Universität Zürich, \\ CH-8057 Zurich, Switzerland \\ c Department of Physics, University Of Alberta, \\ Edmonton, AB T6G 2J1, Canada \\ ${ }^{\mathrm{d}}$ Institute for Nuclear Research of Russian Academy of Sciences, \\ 117312 Moscow, Russia
}

\begin{abstract}
We evaluate the two-loop QED corrections to the Bhabha scattering cross section which involve the vacuum polarization by heavy fermions of arbitrary mass $m_{f} \gg m_{e}$. The results are valid for generic values of the Mandelstam invariants $s, t, u \gg m_{e}^{2}$.
\end{abstract}

PACS numbers: 11.15.Bt, 12.20.Ds

\footnotetext{
${ }^{*}$ Email: Roberto.Bonciani@ific.uv.es

${ }^{\dagger}$ Email: Andrea.Ferroglia@physik.unizh.ch

${ }^{\ddagger}$ Email: apenin@phys. ualberta.ca
} 


\section{Introduction}

Electron-positron Bhabha scattering [1] plays a special role in particle phenomenology. It provides a very efficient tool for luminosity determination at electron-positron colliders and thus it is crucial for extracting physics from the experimental data. Small angle Bhabha scattering is particularly effective as a luminosity monitor at the high energy colliders such as LEP and the future International Linear Collider (ILC) [2,3]. The large angle Bhabha scattering is used to measure the luminosity at colliders operating at a center of mass energy $\sqrt{s}$ of a few GeV, such as BABAR/PEP-II, BELLE/KEKB, BES/BEPC, KLOE/DA $\Phi$ NE, and VEPP-2M [4]. The large angle Bhabha scattering will also be employed in order to disentangle the luminosity spectrum at ILC $[5,6]$. Since the accuracy of the theoretical evaluation of the Bhabha cross section directly affects the luminosity determination, remarkable efforts have been devoted to the study of the radiative corrections to this process (see [2] for an extensive list of references). QED contributions dominate the radiative corrections to the large angle scattering at intermediate energies 1-10 GeV and to the small angle scattering also at higher energies. The calculation of the QED radiative corrections to the Bhabha cross section is among the classical problems of perturbative quantum field theory and has a long history. The first-order corrections are well known (see [7] and references therein). However, to match the impressive experimental accuracy reached at colliders, the complete secondorder QED effects have to be taken into account. The evaluation of the two-loop virtual corrections is the main problem of the second-order analysis. The two-loop QED corrections can be divided into three main categories: (i) the corrections involving the electron vacuum polarization i.e. with at least one closed electron loop, (ii) the pure photonic corrections, and (iii) the corrections involving the vacuum polarization by heavy flavors (leptons or quarks) of mass $m_{f} \gg m_{e}$. Type (i) corrections have been evaluated in [8] including the full dependence on the electron mass $m_{e}$. For the virtual corrections of type (ii) the full dependence on the electron mass is known with the exception of the two-loop box diagrams [11]. ${ }^{4}$ At the same time, the complete result for the pure photonic corrections has been obtained in [12] in the leading order of the small electron mass expansion through the infrared matching to the massless approximation [13]. ${ }^{5}$ It is sufficient for all the phenomenological applications and has been recently confirmed within a slightly different framework [16]. For the heavy-flavor contribution the result is available only in the limit $m_{f}^{2} \ll s, t, u[16,17]$. This condition, however, does not hold for $\tau$-lepton, $c$ - and $b$-quark in the practically interesting energy range of about a few $\mathrm{GeV}$ as well as for the top quark at the typical ILC energies $500 \mathrm{GeV} \lesssim \sqrt{s} \lesssim 1000 \mathrm{GeV}$.

In this letter we consider the two-loop heavy-flavor contribution retaining the full dependence on $m_{f}^{2} / s$. In the next section we outline the structure of the corrections and the calculation method. In Sect. 3 we present the complete numerical result for the correction in two phenomenologically interesting cases. Sect. 4 contains our conclusions. The expansion

\footnotetext{
${ }^{4}$ Partial results for the massive Bhabha scattering two-loop box diagrams are discussed in $[9,10]$.

${ }^{5}$ The terms enhanced by logarithms of the electron mass have been derived in this approximation in $[14,15]$.
} 
of the result in the limits $m_{f}^{2} \ll s$ and $m_{f}^{2} \gg s$ is given in the Appendix in analytic form.

\section{Structure of the Radiative Corrections and Calcula- tion Method}

We consider the phenomenologically interesting kinematical region $s, t, u \sim m_{f}^{2} \gg m_{e}^{2}$, where all the terms suppressed by the electron mass can be neglected. The perturbative expansion for the Bhabha cross section in the fine structure constant $\alpha$ is defined as follows

$$
\frac{\mathrm{d} \sigma}{\mathrm{d} \Omega}=\sum_{n=0}^{\infty}\left(\frac{\alpha}{\pi}\right)^{n} \delta^{(n)} \frac{\mathrm{d} \sigma^{(0)}}{\mathrm{d} \Omega}
$$

where $\delta^{(n)}$ is the correction factor, with $\delta^{(0)}=1$. The leading order differential cross section in the small electron mass approximation takes the form

$$
\frac{\mathrm{d} \sigma^{(0)}}{\mathrm{d} \Omega}=\frac{\alpha^{2}}{s}\left(\frac{1-x+x^{2}}{x}\right)^{2}+\mathcal{O}\left(m_{e}^{2} / s\right)
$$

where $x=(1-\cos \theta) / 2$ and $\theta$ is the scattering angle. The virtual corrections are infrared divergent. These soft divergencies are canceled in the inclusive cross section when one adds the photonic bremsstrahlung [18]. The standard approach to deal with the bremsstrahlung is to split it into a soft part, which accounts for the emission of the photons with the energy below a given cutoff $\varepsilon_{c u t} \ll m_{e}$, and a hard part corresponding to the emission of the photons with the energy larger than $\varepsilon_{c u t}$. The infrared finite hard part is then computed numerically using Monte-Carlo methods with physical cuts dictated by the experimental setup. At the same time, the soft part is computed analytically and combined with the virtual corrections ensuring the cancellation of the soft divergencies in Eq. (1). We regulate all the soft divergencies by dimensional regularization in $d=4-2 \varepsilon$ space-time dimensions.

The first-order heavy-flavor contribution to the cross section reads

$$
\delta^{(1)}=\frac{1}{1-x+x^{2}}\left\{x(2 x-1) \operatorname{Re}\left[\Pi_{f}^{(1)}(\rho)\right]+(2-x) \operatorname{Re}\left[\Pi_{f}^{(1)}(\rho / x)\right]\right\}
$$

where

$$
\Pi_{f}^{(1)}(\rho)=\frac{Q_{f}^{2} N_{c}}{3}\left[-\frac{5}{3}+4 \rho-(1+2 \rho) \sqrt{1-4 \rho}\left(2 \operatorname{arctanh} \sqrt{\frac{1}{1-4 \rho}}+i \pi \theta\left(\frac{1}{\rho}-4\right)\right)\right]
$$

is the well known one-loop vacuum polarization function, $\rho=m_{f}^{2} / s, Q_{f}$ is the heavy-flavor electric charge, and the number of colors $N_{c}$ is 1 for leptons and 3 for quarks. We work within the standard on-shell QED renormalization scheme. The second-order contribution can be split in the sum of two terms

$$
\delta^{(2)}=\delta_{v v}^{(2)}+\delta_{s}^{(1)} \delta^{(1)}
$$




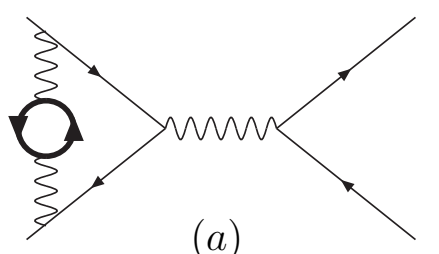

$(a)$

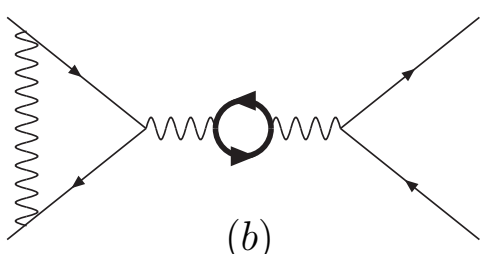

(b)

Figure 1: The two-loop diagrams associated with the logarithmic dependence of the corrections to the Bhabha scattering amplitude on $m_{e}$. Actually the diagram $(a)$ is free of electron mass logarithms. The bold arrow circle corresponds to the heavy-flavor vacuum polarization.

which correspond to the two-loop virtual correction ${ }^{6}$ and the one-loop correction to the single soft photon emission which factorizes into the product of the first-order contributions [19].

The calculation of the virtual corrections is a highly nontrivial problem since it involves the two-loop four-point Feynman integrals that depend on four independent mass parameters. However, the calculation is significantly simplified in the small electron mass limit. Eq. (5) can be rewritten according to the asymptotic dependence on $m_{e}$

$$
\delta^{(2)}=\delta_{1}^{(2)} \ln \left(\frac{s}{m_{e}^{2}}\right)+\delta_{0}^{(2)}+\mathcal{O}\left(m_{e}^{2} / s\right) .
$$

The logarithmic term in Eq. (6) is a remnant of the collinear divergence which is regulated by the electron mass. The quantities $\delta_{1}^{(2)}$ and $\delta_{0}^{(2)}$ in Eq. (6) depend on $s, t$, and $m_{f}$ only. The collinear divergencies and hence the singular dependence of the corrections on $m_{e}$ have a peculiar structure which was extensively studied in the context of QCD. In particular, the collinear divergencies factorize and can be absorbed in the external field renormalization [20]. This property is crucial for our analysis because it allows us to perform the most difficult part of the calculation with a strictly massless electron. Indeed, due to the factorization, the singular dependence on $m_{e}$ is the same for the Bhabha amplitude and (the square of) the vector form factor [12]. This attributes the total logarithmic corrections to the twoloop Bhabha scattering amplitude to the diagrams shown in Fig. 1. However, due to the renormalization condition the vacuum polarization does not change the photon propagator near the mass shell where the collinear divergencies are located. As a result, the diagram $(a)$ is infrared finite even for $m_{e}=0$ and the singular terms are entirely contained in the oneparticle reducible diagram $(b)$. In calculating the cross section one must take into account also the contributions coming from the interference of the one-loop corrections to the amplitude and the corresponding soft emission; both contributions have a factorized form. Thus it is straightforward to obtain the coefficient of the logarithmic term in Eq. (6) which reads

$$
\delta_{1}^{(2)}=\left[4 \ln \left(\frac{\varepsilon_{c u t}}{\varepsilon}\right)+3\right] \delta^{(1)},
$$

\footnotetext{
${ }^{6}$ We do not consider the trivial correction given by two heavy-fermion loop insertions.
} 
where $\varepsilon=\sqrt{s} / 2$. At the same time the sum of the one-particle irreducible diagrams has a regular behavior in the small electron mass limit and can be computed with $m_{e}=0$. Let us emphasize that this property in general holds only for the sum of the diagrams. The individual diagrams computed in a covariant gauge do exhibit the collinear divergencies for $m_{e}=0$. This however does not pose any additional problem since we work in dimensional regularization. In this case the collinear divergencies show up as the extra poles in $\varepsilon$ which are not related to the soft emission and disappear in the sum of the one-particle irreducible diagrams. Thus all the "true" two-loop diagrams contribute only to the non logarithmic term in Eq. (6) which can be written as follows

$$
\delta_{0}^{(2)}=-4\left[1+\ln \left(\frac{1-x}{x}\right)\right] \ln \left(\frac{\varepsilon_{c u t}}{\varepsilon}\right) \delta^{(1)}+Q_{f}^{2} N_{c}\left(\frac{x}{1-x+x^{2}}\right)^{2} f(\rho, x),
$$

where the first term is determined by the soft emission and $f(\rho, x)$ is a function of two dimensionless variables: $\rho=m_{f}^{2} / s$ and $x=-t / s$. The two-loop problem with massless electron falls it the same complexity class as the one considered in Ref. [21]. The most difficult part of the calculation is represented, as expected, by the evaluation of the two-loop box graphs. By employing the Laporta Algorithm [22] to identify the set of master integrals (MI), it is possible to conclude that just two of the MI necessary for this calculation correspond to four point functions. These two are the only unknown ones among the MIs appearing in the calculation. They were evaluated by means of the differential equation method [23] and expressed in terms of Harmonic Polylogarithms [24], and suitable generalizations of the latter [25]. The technical aspects of the calculation are going to be discussed in detail in [26]. Finally, the result for the function $f(\rho, x)$ can be expressed in closed analytic form in terms of one- and two-dimensional generalized harmonic polylogarithms of maximum weight three.

\section{$3 \quad$ Results and Numerical Estimates}

The complete analytical result is rather lengthy and will be published elsewhere. In this letter we focus on the numerical impact of the correction in the phenomenologically important cases and we present the approximate analytical expression in different limits.

As the first application, we consider the Bhabha scattering at the energy of $1 \mathrm{GeV}$ which plays a crucial role in the determination of the hadronic vacuum polarization contribution to the muon anomalous magnetic moment $[27,28]$. The second-order contributions $(\alpha / \pi)^{2} \delta^{(2)}$ of heavy leptons and quarks to the differential cross section are plotted as function of the scattering angle in Figs. 2 and 3 for $\sqrt{s}=1 \mathrm{GeV}$, which is the center-of-mass energy of the KLOE experiment at DA $\Phi$ NE. All the terms involving a power of the logarithm $\ln \left(\varepsilon_{c u t} / \varepsilon\right)$ are excluded from the numerical estimates because the corresponding contribution critically depends on the event selection algorithm and cannot be unambiguously estimated without imposing specific cuts on the photon bremsstrahlung. Note that the energy under consideration is sufficiently below the quarkonium threshold and the heavy-quarks can be treated perturbatively. The contributions of the $\tau$-lepton, $c$ and $b$ quarks are suppressed 


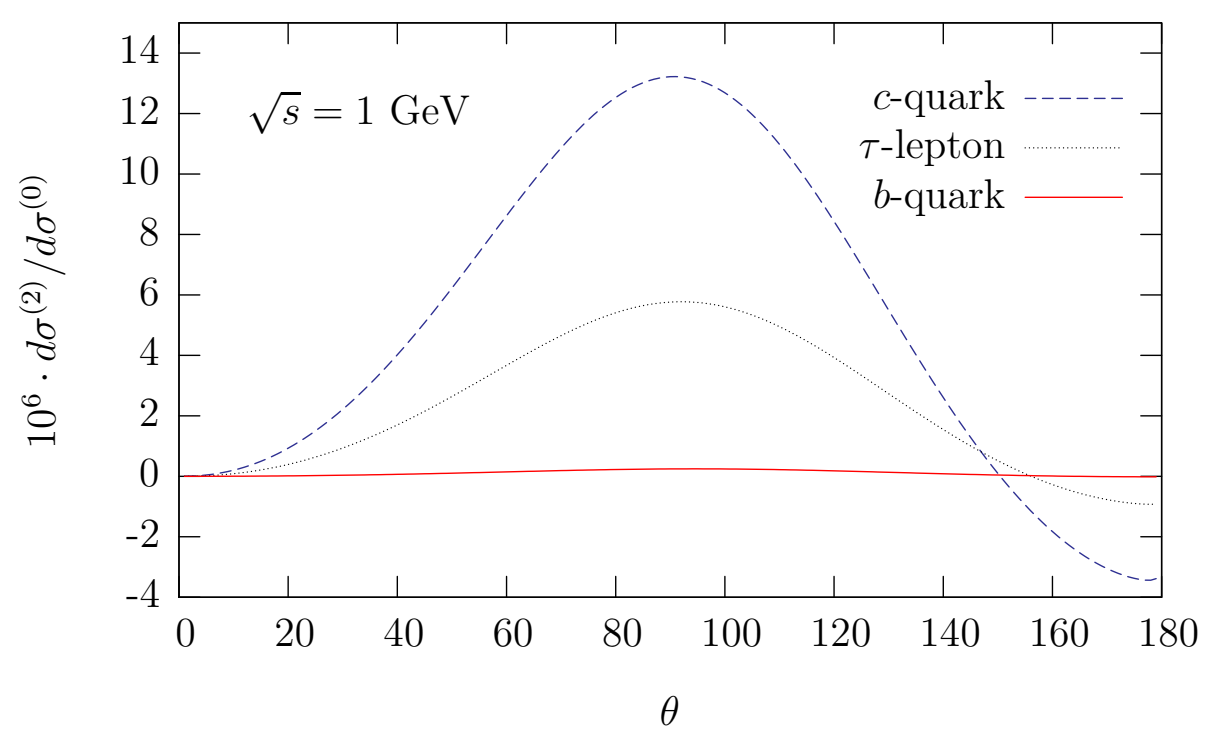

Figure 2: Two-loop corrections to the Bhabha scattering differential cross section at $\sqrt{s}=1 \mathrm{GeV}$ due to a closed loop of $\tau$-lepton (dotted line), $c$-quark (dashed line) and $b$-quark (solid line) for $m_{c}=1.25 \mathrm{GeV}$ and $m_{b}=4.7 \mathrm{GeV}$.

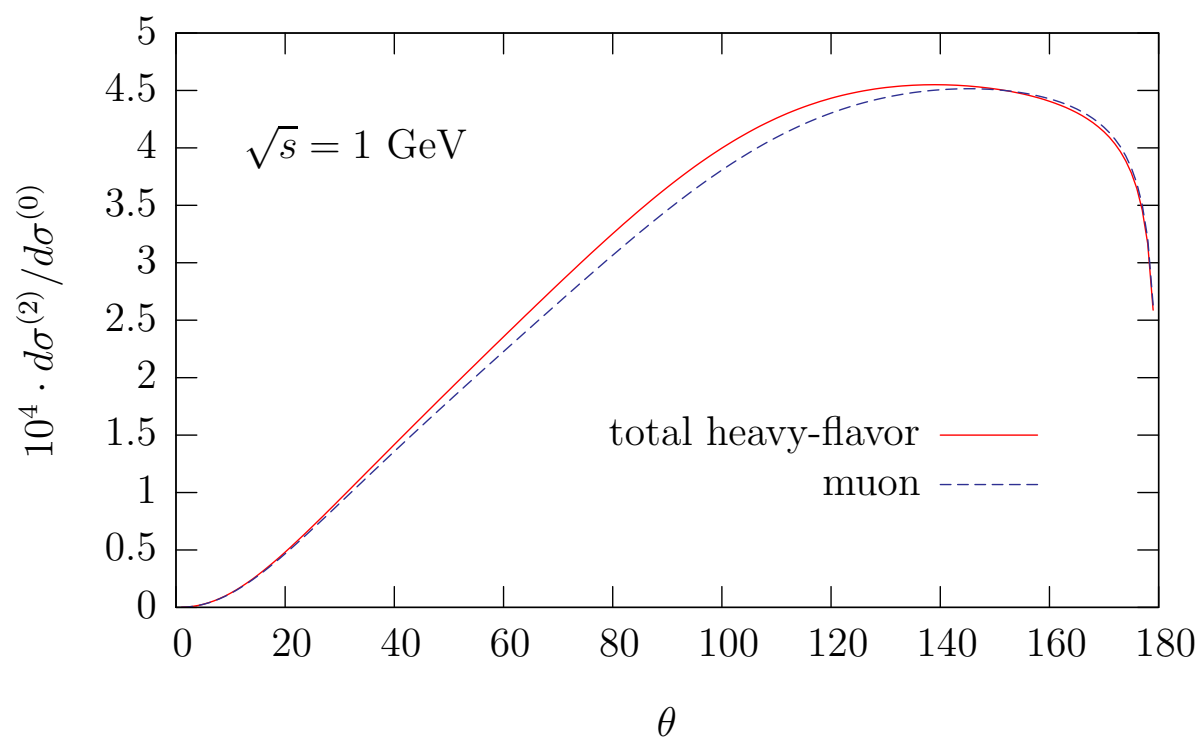

Figure 3: Two-loop corrections to the Bhabha scattering differential cross section at $\sqrt{s}=1 \mathrm{GeV}$ due to a closed loop of muon (dashed line). The solid line represents the sum of the contributions of the muon, $\tau$-lepton, $c$-quark and $b$-quark. 


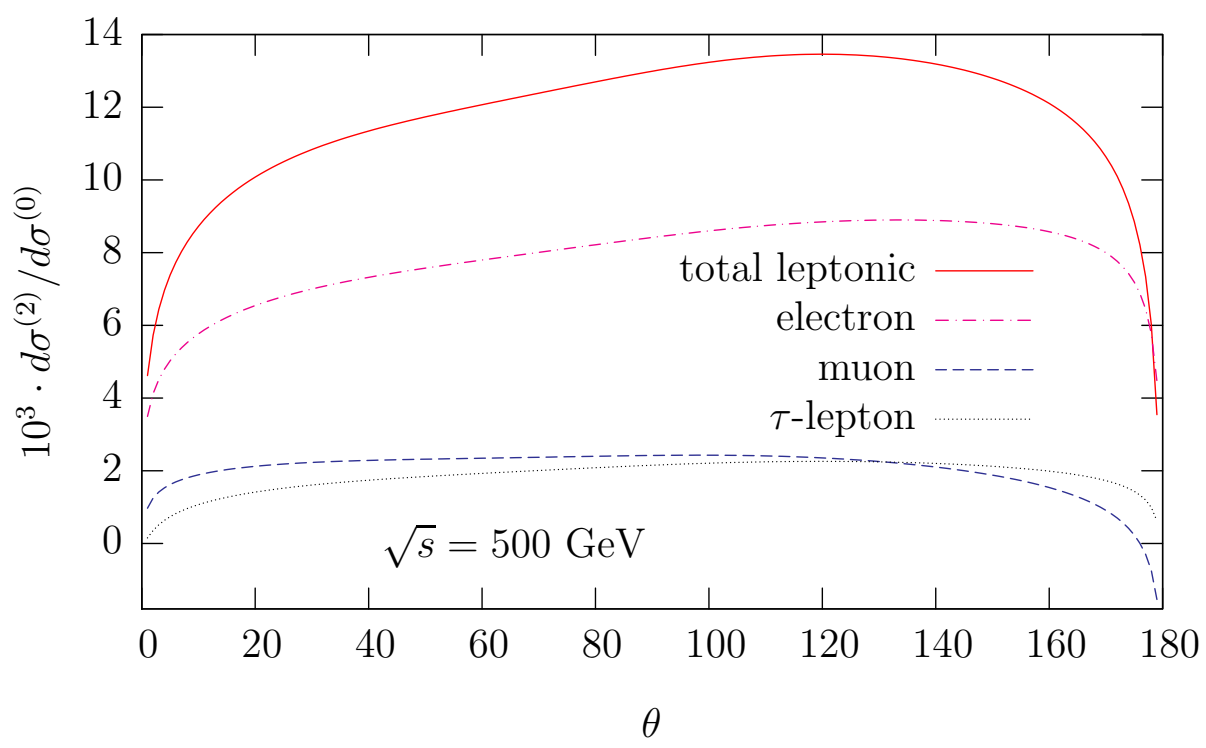

Figure 4: Two-loop leptonic corrections to the Bhabha scattering differential cross section at $\sqrt{s}=$ $500 \mathrm{GeV}$. The dash-dotted line represents the electron contribution including the soft-pair radiation. The dashed and dotted lines represent the contributions of muon and $\tau$-lepton, respectively. The solid line is the sum of the three contributions.

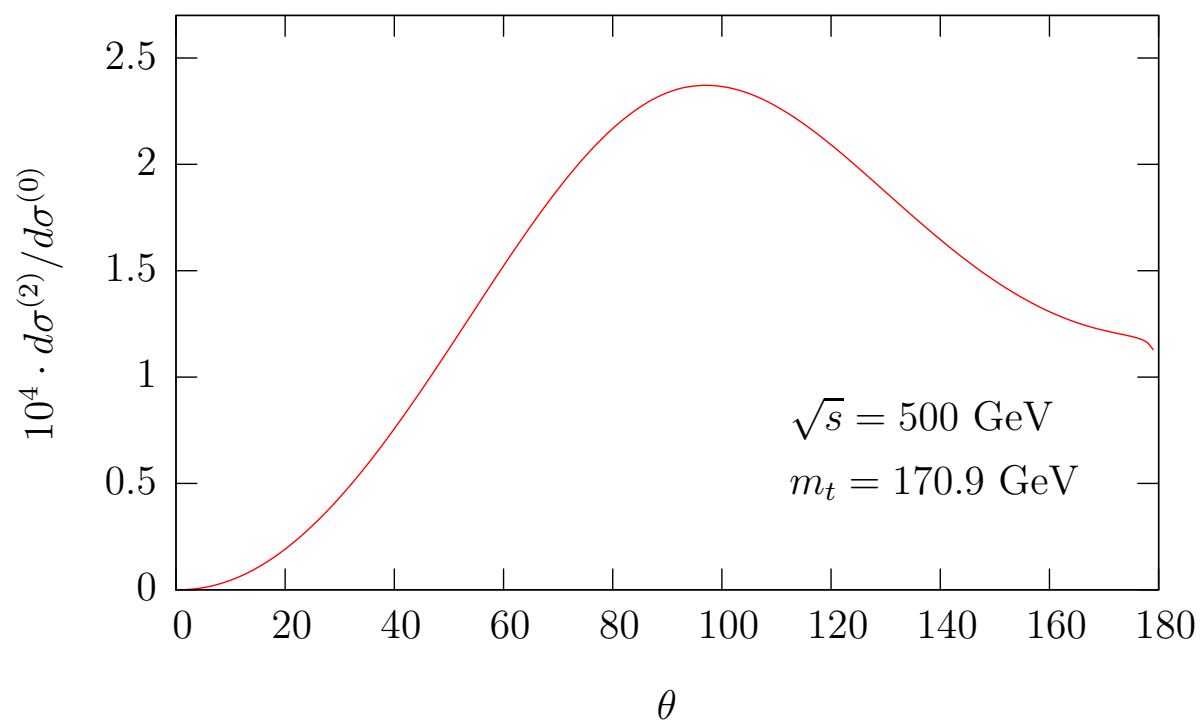

Figure 5: Two-loop corrections to the Bhabha scattering differential cross section at $\sqrt{s}=500 \mathrm{GeV}$ due to a closed loop of top quark for $m_{t}=170.9 \mathrm{GeV}$. 


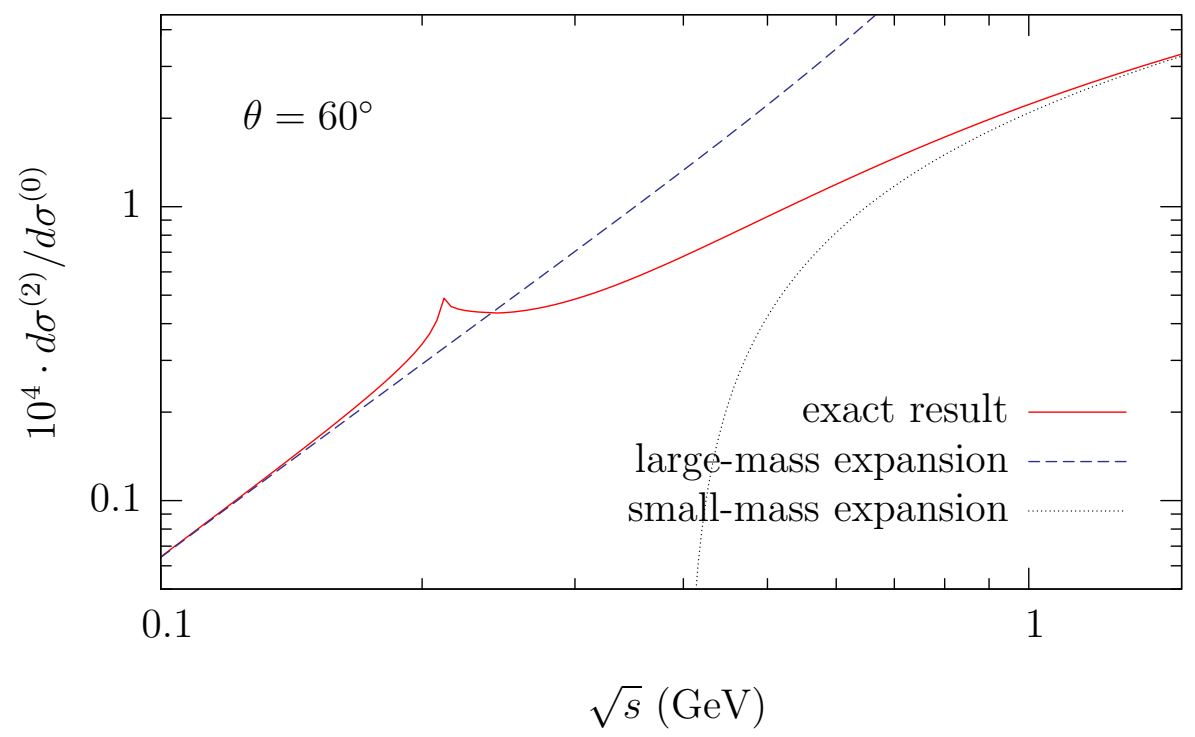

Figure 6: Two-loop corrections to the Bhabha scattering differential cross section at $\theta=60^{\circ}$ due to a closed loop of muon. The solid line represents the exact result. The dashed and dotted lines represent the results of the large-mass expansion (Eq. (13)) and small-mass expansion (Eq. (10)), respectively.

with respect to the muon at least by one order of magnitude and they are given separately in Fig. 2. Thus the total heavy-flavor contribution is dominated by the muon loop and it reaches 0.45 permille in magnitude at $\theta \sim 140^{\circ}$, see Fig. 3.

Let us now discuss Bhabha scattering at high energies characteristic to the ILC. We consider only the contributions of the leptons and the top quark because the lighter quarks need a special treatment due to hadronization effects [29]. In Fig. 4 we present the plots for the contributions of muon, $\tau$-lepton, and electron (including the soft electron-positron pair emission) at $\sqrt{s}=500 \mathrm{GeV}$. The total leptonic correction reaches $1.3 \%$ at $\theta \sim 120^{\circ}$ and it is comparable with the pure photonic term [12]. The contribution of top quark is plotted in Fig. 5. It is significantly smaller than the leptonic one.

The analytical structure of the result becomes much simpler in the small-mass limit $m_{f}^{2} \ll s$ and the large-mass limit $m_{f}^{2} \gg s$. The corresponding expansions of the function $f(\rho, x)$ at $\rho \rightarrow 0$ and $\rho \rightarrow \infty$ are given in the Appendix. Both expansions break down near the threshold $\sqrt{s} \sim 2 m_{f}$. In Fig. 6 the muon contribution at the fixed scattering angle $\theta=60^{\circ}$ is plotted as function of $\sqrt{s}$ in the threshold region around $2 m_{\mu} \approx 216 \mathrm{MeV}$. In this plot we present the exact result along with the first nonvanishing terms of the small and large mass expansions, Eqs. $(10,13)$. We observe that the asymptotic results fail to approximate the exact one with $10 \%$ accuracy in the interval $1.7 \lesssim \sqrt{s} / m_{f} \lesssim 10$. In particular they are completely useless for the description of the top quark contribution at the typical energies of the ILC. 


\section{Summary}

To conclude, we have derived the two-loop radiative corrections to Bhabha scattering due to the heavy-flavor vacuum polarization in the leading order of the small electron mass expansion and for any ratio of the heavy-fermion mass to the energy of the process. This completes the calculation of the QED part of the two-loop corrections. We have analyzed the phenomenological impact of our result in two different experimental setups. For $e^{+} e^{-}$ colliders operating at the energies of a few $\mathrm{GeV}$, the correction is dominated by the virtual muon loop and reaches 0.45 permille at large scattering angles. At the typical ILC energies the $\tau$-lepton and muon loops result in the comparable contributions of about two permille so that the total leptonic correction amounts up to $1.3 \%$. The contribution of the top quark is smaller and at $\sqrt{s}=500 \mathrm{GeV}$ reaches approximately 0.25 permille in magnitude.

\section{Acknowledgements}

We are grateful to J. Vermaseren and D. Maitre for their kind assistance in the use of FORM [30], and of the Mathematica packages HPL and HypExp [31]. A. F. and R. B. would like to thank S. Actis for useful discussions. R. B. would like to thank D. Greynat for useful discussions about Mellin-Barnes [32], the Galileo Galilei Institute for Theoretical Physics for the hospitality and the INFN for partial support during the completion of this work. The work of R. B. was partially supported by Ministerio de Educación y Ciencia (MEC) under grant FPA2004-00996, Generalitat Valenciana under grant GVACOMP2007-156, European Commission under the grant MRTN-CT-2006-035482 (FLAVIAnet), and MEC-INFN agreement. The work of A. F. was supported in part by the Swiss National Science Foundation (SNF) under contract 200020-117602. The work of A. P. was supported in part by BMBF Grant No. 05HT4VKA/3 and Sonderforschungsbereich Transregio 9.

\section{Appendix}

The small-mass expansion of the function $f(\rho, x)$ is of the following form

$$
f(\rho, x)=\sum_{n=0}^{\infty} \rho^{n} f_{n}(\rho, x),
$$

where $f_{n}(\rho, x)$ depend on $\rho$ only logarithmically. For the leading term we obtain

$$
\begin{aligned}
f_{0}(\rho, x) & =\frac{\left(x^{2}-x+1\right)^{2}}{x^{2}}\left\{\frac{1}{9} \ln ^{3}(\rho)+\ln ^{2}(\rho)\left[\frac{1}{3} \ln (1-x)+\frac{19}{18}-\frac{1}{3} \ln (x)\right]\right. \\
& \left.+\ln (\rho)\left[\frac{191}{27}+\frac{8}{3} \operatorname{Li}_{2}(x)\right]+\frac{40}{9} \operatorname{Li}_{2}(x)+\frac{1165}{81}\right\}-\ln (\rho)[ \\
& +\frac{32 x^{4}-46 x^{3}+33 x^{2}+8 x-4}{6 x^{2}} \zeta(2)-\frac{\left(x^{2}-x+1\right)\left(4 x^{2}-7 x+4\right)}{6 x^{2}} \ln (1-x)^{2}
\end{aligned}
$$




$$
\begin{aligned}
& -\frac{20 x^{4}-31 x^{3}+60 x^{2}-31 x+20}{18 x^{2}} \ln (1-x)+\frac{20 x^{4}-67 x^{3}+141 x^{2}-112 x+74}{18 x^{2}} \ln (x) \\
& \left.+\frac{8 x^{4}-x^{3}-15 x^{2}+17 x-4}{12 x^{2}} \ln (x)^{2}-\frac{(2 x-1)\left(4 x^{3}-3 x^{2}+4\right)}{6 x^{2}} \ln (x) \ln (1-x)\right] \\
& +\frac{(2 x-1)\left(x^{2}-x+1\right)}{3 x} \zeta(3)-\frac{(x-1)^{2}\left(x^{2}-x+1\right)}{9 x^{2}} \ln ^{3}(1-x) \\
& -\frac{196 x^{4}-311 x^{3}+258 x^{2}+13 x-38}{18 x^{2}} \zeta(2)-\frac{2\left(2 x^{4}-9 x^{3}+16 x^{2}-11 x+4\right)}{3 x^{2}} \ln (1-x) \zeta(2) \\
& +\frac{12 x^{4}-20 x^{3}-x^{2}+24 x-4}{6 x^{2}} \ln (x) \zeta(2)+\frac{2\left(1-x^{2}\right)\left(x^{2}-x+1\right)}{3 x^{2}} \ln (1-x) \operatorname{Li}_{2}(x) \\
& +\frac{7\left(16 x^{4}-23 x^{3}+48 x^{2}-23 x+16\right)}{54 x^{2}} \ln (1-x)+\frac{20 x^{4}-58 x^{3}+81 x^{2}-58 x+20}{18 x^{2}} \ln ^{2}(1-x) \\
& -\frac{4 x^{3}-6 x^{2}+7 x-4}{12 x} \ln (x) \ln ^{2}(1-x)+\frac{40 x^{4}-50 x^{3}+9 x^{2}+37 x-20}{18 x^{2}} \ln (x) \ln (1-x) \\
& -\frac{x^{4}-3 x^{3}+4 x^{2}-x+1}{3 x^{2}} \ln ^{2}(x) \ln (1-x)+\frac{4 x^{4}-2 x^{3}-22 x^{2}+31 x-4}{36 x^{2}} \ln ^{3}(x) \\
& -\frac{20 x^{4}+8 x^{3}-84 x^{2}+92 x-55}{18 x^{2}} \ln 2(x)-\frac{\left(x^{2}-x+1\right)\left(2 x^{2}-7 x+12\right)}{3 x^{2}} \ln (x) \operatorname{Li}_{2}(x) \\
& -\frac{112 x^{4}-449 x^{3}+1011 x^{2}-836 x+562}{54 x^{2}} \ln (x) \\
& +\frac{2\left(1-x^{2}\right)\left(x^{2}-x+1\right)}{3 x^{2}} \operatorname{Li}_{3}(1-x)+\frac{\left(x^{2}-x+1\right)\left(2 x^{2}-3 x+4\right)}{3 x^{2}} \operatorname{Li}_{3}(x) \\
& \left.\left.-\left(Q_{f}^{2}-1\right) \frac{1}{2} \ln (\rho)\right)-\frac{2-x}{4} \ln (x)\right],
\end{aligned}
$$

where $Q_{f}$ is the charge of the heavy fermion in units of the electron charge. Eq. (10) is in agreement with the result of Refs. $[16,17]$. The next-to-leading term is new and reads

$$
\begin{aligned}
f_{1}(\rho, x) & =\frac{2(x-1)\left(x^{2}-x+1\right)\left(2 x^{2}+x+2\right)}{x^{3}}\left[\ln ^{2}(\rho)+4 \operatorname{Li}_{2}(x)+12\right] \\
& +\ln (\rho)\left\{\frac{(x-1)}{x^{3}}\left(2 x^{4}-5 x^{3}+5 x^{2}-5 x+2\right)-\frac{2\left(x^{2}-x+1\right)}{x^{3}}\left[\left(2 x^{3}-x^{2}+2 x-4\right) \ln (x)\right.\right. \\
& \left.\left.-(x-1)\left(2 x^{2}+x+2\right) \ln (1-x)\right]\right\}-\frac{40 x^{5}-54 x^{4}+50 x^{3}-17 x^{2}-12 x+8}{x^{3}} \zeta(2) \\
& +\frac{(x-1)}{2 x^{3}}\left[2\left(12 x^{4}-5 x^{3}+13 x^{2}-5 x+12\right)+\left(8 x^{4}-6 x^{3}+9 x^{2}-6 x+8\right) \ln (1-x)\right] \ln (1-x) \\
& -\left[\frac{12 x^{5}-21 x^{4}+26 x^{3}-26 x^{2}+21 x-14}{x^{3}}-\frac{8 x^{5}-10 x^{4}+8 x^{3}-x^{2}-6 x+4}{x^{3}} \ln (1-x)\right. \\
& \left.+\frac{8 x^{5}-7 x^{4}-x^{3}+12 x^{2}-15 x+8}{2 x^{3}} \ln (x)\right] \ln (x)+\frac{3\left(Q_{f}^{2}-1\right)}{x^{3}}\left[\left(2-3 x+4 x^{2}-4 x^{3}\right.\right. \\
& \left.\left.+3 x^{4}-2 x^{5}\right) \ln (\rho)-\left(2-3 x+3 x^{2}-x^{3}\right) \ln (x)\right] \cdot
\end{aligned}
$$


The expansion in the large-mass limit takes the form

$$
f(\rho, x)=\sum_{n=0}^{\infty} \rho^{-n} \bar{f}_{n}(\rho, x),
$$

where the leading $n=0$ term vanishes because of the renormalization condition and $\bar{f}_{n}(\rho, x)$ depend on $\rho$ only logarithmically. For the next-to-leading term we obtain

$$
\begin{aligned}
\bar{f}_{1}(\rho, x)= & \frac{955 x^{3}-3926 x^{2}+3926 x-955}{1350 x}-\frac{12 x^{3}-19 x^{2}+14 x-6}{10 x} \zeta(2) \\
& +\frac{3 x^{3}+x^{2}-x-3}{30 x} \ln (1-x)+\frac{2 x^{3}-5 x^{2}+5 x-2}{20 x} \ln ^{2}(1-x) \\
& +\frac{5 x^{3}-22 x^{2}+22 x-5}{30 x} \ln (\rho)-\frac{20 x^{3}-78 x^{2}+93 x-58}{90 x} \ln x \\
& +\frac{12 x^{3}-19 x^{2}+14 x-6}{30 x} \ln x \ln (1-x)-\frac{1}{60}\left(6 x^{2}-x-4\right) \ln ^{2} x \\
& +\frac{4\left(x^{3}-2 x^{2}+2 x-1\right)}{5 x} \operatorname{Li}_{2}(x)-\left(Q_{f}^{2}-1\right) \frac{41\left(x^{3}-2 x^{2}+2 x-1\right)}{54 x} .
\end{aligned}
$$

\section{References}

[1] J.H.Bhabha, Proc.Roy.Soc. 154A, 195 (1935).

[2] S. Jadach et al. in G. Altarelli, T. Sjöstrand and F. Zwirner (eds.), Physics at LEP2, CERN-96-01, [arXiv:hep-ph/9602393].

[3] G. Montagna, O. Nicrosini, and F. Piccinini, Riv. Nuovo Cim. 21N9, 1 (1998) [arXiv:hep-ph/9802302].

[4] C. M. Carloni Calame, C. Lunardini, G. Montagna, O. Nicrosini, and F. Piccinini, Nucl. Phys. B584, 459 (2000) [arXiv:hep-ph/0003268].

G. Balossini, C. M. Carloni Calame, G. Montagna, O. Nicrosini and F. Piccinini, Nucl. Phys. B 758, 227 (2006) [arXiv:hep-ph/0607181].

[5] N. Toomi, J. Fujimoto, S. Kawabata, Y. Kurihara, and T. Watanabe, Phys. Lett. B 429, 162 (1998).

[6] J. A. Aguilar-Saavedra et al. [ECFA/DESY LC Physics Working Group], arXiv:hep-ph/0106315.

[7] M. Consoli, Nucl. Phys. B 160208 (1979).

M. Bohm, A. Denner and W. Hollik, Nucl. Phys. B 304687 (1988).

F. A. Berends and R. Kleiss, Nucl. Phys. B 228, 537 (1983).

M. Caffo, R. Gatto and E. Remiddi, Nucl. Phys. B 252, 378 (1985). 
[8] R. Bonciani, P. Mastrolia and E. Remiddi, Nucl. Phys. B 676, 399 (2004) [arXiv:hep-ph/0307295].

R. Bonciani, A. Ferroglia, P. Mastrolia, E. Remiddi and J. J. van der Bij, Nucl. Phys. B 701121 (2004) [arXiv:hep-ph/0405275]. Nucl. Phys. B716, 280 (2005) [arXiv:hep-ph/0411321].

[9] G. Heinrich and V. A. Smirnov, Phys. Lett. B 598, 55 (2004) [arXiv:hep-ph/0406053].

[10] V. A. Smirnov, Phys. Lett. B 524, 129 (2002) [arXiv:hep-ph/0111160].

[11] R. Bonciani and A. Ferroglia, Phys. Rev. D 72, 056004 (2005) [arXiv:hep-ph/0507047].

[12] A. A. Penin, Phys. Rev. Lett. 95, 010408 (2005) [arXiv:hep-ph/0501120]; Nucl. Phys. B734, 185 (2006) [arXiv:hep-ph/0508127].

[13] Z. Bern, L. Dixon, and A. Ghinculov, Phys. Rev. D 63, 053007 (2001) [arXiv:hep-ph/0010075].

[14] A. B. Arbuzov, E. A. Kuraev and B. G. Shaikhatdenov, Mod. Phys. Lett. A 13, 2305 (1998) [arXiv:hep-ph/9806215].

[15] E. W. N. Glover, J. B. Tausk and J. J. Van der Bij, Phys. Lett. B 51633 (2001) [arXiv:hep-ph/0106052].

[16] T. Becher and K. Melnikov, JHEP 0706, 084 (2007) [arXiv:0704.3582 [hep-ph]].

[17] S. Actis, M. Czakon, J. Gluza and T. Riemann, arXiv:0704.2400 [hep-ph].

[18] T. Kinoshita, J. Math. Phys. 3, 650 (1962).

T. D. Lee and M. Nauenberg, Phys. Rev. 133, B1549 (1964).

[19] D. R. Yennie, S. C. Frautschi and H. Suura, Annals Phys. 13, 379 (1961).

[20] J. Frenkel and J. C. Taylor, Nucl. Phys. B 116, 185 (1976).

[21] R. Bonciani, P. Mastrolia and E. Remiddi, Nucl. Phys. B 661, 289 (2003) [arXiv:hep-ph/0301170] [Erratum-ibid. B 702, 359 (2004)]. Nucl. Phys. B 690138 (2004) [arXiv:hep-ph/0311145].

R. Bonciani, A. Ferroglia, P. Mastrolia, E. Remiddi and J. J. van der Bij, Nucl. Phys. B 681, 261 (2004) [Erratum-ibid. B 702, 364 (2004)] [arXiv:hep-ph/0310333].

M. Czakon, J. Gluza and T. Riemann, Phys. Rev. D 71, 073009 (2005) [arXiv:hep-ph/0412164]. Nucl. Phys. B 751, 1 (2006) [arXiv:hep-ph/0604101].

[22] S. Laporta and E. Remiddi, Phys. Lett. B 379 (1996) 283. [arXiv:hep-ph/9602417];

S. Laporta, Int. J. Mod. Phys. A 15 (2000) 5087 [arXiv:hep-ph/0102033]. 
[23] A. V. Kotikov, Phys. Lett. B 254 (1991) 158; Phys. Lett. B 259 (1991) 314; Phys. Lett. B 267 (1991) 123;

E. Remiddi, Nuovo Cim. A 110 (1997) 1435 [arXiv:hep-th/9711188];

M. Caffo, H. Czyz, S. Laporta and E. Remiddi, Acta Phys. Polon. B 29 (1998) 2627; [arXiv:hep-th/9807119]; Nuovo Cim. A 111 (1998) 365. [arXiv:hep-th/9805118].

M. Argeri and P. Mastrolia, Int. J. Mod. Phys. A 22, 4375 (2007) [arXiv:0707.4037 [hep-ph]].

[24] A.B.Goncharov, Math. Res. Lett. 5 (1998), 497-516;

D. J. Broadhurst, Eur. Phys. J. C 8 (1999) 311. [arXiv:hep-th/9803091];

E. Remiddi and J. A. M. Vermaseren, Int. J. Mod. Phys. A 15, 725 (2000) [arXiv:hep-ph/9905237].

[25] U. Aglietti and R. Bonciani, Nucl. Phys. B 698, 277 (2004) [arXiv:hep-ph/0401193].

[26] R. Bonciani, A. Ferroglia, and A. A. Penin, in preparation.

[27] A. Aloisio et al. [KLOE Collaboration], Phys. Lett. B 606, 12 (2005) [arXiv:hep-ex/0407048].

[28] S. Eidelman [CMD-2 and SND Collaborations], PoS HEP2005, 114 (2006).

[29] B. A. Kniehl, M. Krawczyk, J. H. Kuhn, and R. G. Stuart, Phys. Lett. B 209, 337 (1988).

[30] J.A.M. Vermaseren, Symbolic Manipulation with FORM, Version 2, CAN, Amsterdam, 1991; "New features of FORM" [arXiv:math-ph/0010025].

[31] D. Maitre, arXiv:hep-ph/0703052. Comput. Phys. Commun. 174, 222 (2006) [arXiv:hep-ph/0507152].

T. Huber and D. Maitre, Comput. Phys. Commun. 175, 122 (2006) [arXiv:hep-ph/0507094]. arXiv:0708.2443 [hep-ph].

[32] S. Friot, D. Greynat and E. De Rafael, Phys. Lett. B 628, 73 (2005) [arXiv:hep-ph/0505038]. 